\title{
Radiographic Evaluation of Crestal Bone Level Changes for Allografts or Xenografts Placed during Implant Placement: A Retrospective Study
}

\author{
Chiang X Mae ${ }^{1}$, Naresh Y Shetty ${ }^{2}$, Pravinkumar G Patil ${ }^{3}$
}

\begin{abstract}
Aim:The aim of this study was to evaluate initial peri-implant crestal bone level changes when the allografts and xenografts were placed simultaneously during the implant placement.

Materials and methods: This retrospective study was planned with the convenient sampling total of 77 implant sites that were bone grafted either with allografts (group I) or xenografts (group II). Using the periapical radiographs obtained after placement of bone grafts as baseline, the changes in the alveolar crestal bone around the implants were evaluated by comparing periapical radiographs taken at 3 months and 6 or 8 months after the surgery.

Results: The alveolar bone loss at crestal region at the time of placement of bone grafts were $-1.85 \pm 1.26 \mathrm{~mm}$ at the xenograft sites and $-1.75 \pm 1.51 \mathrm{~mm}$ at allograft sites, respectively $(p=0.791)$. At the time of reentry, 3 months after tooth extraction and ridge preservation, the bone dimensions were $1.17 \pm 0.83 \mathrm{~mm}$ for xenograft and $1.00 \pm 1.14 \mathrm{~mm}$ for allograft $(p=0.523)$. At the final reentry, bone-grafted sites were divided into after 6 months and after 8 months postoperatively. After 3 months, the allografts showed lesser bone resorption $(0.9 \pm 0.52 \mathrm{~mm})$ as compared with the xenografts $(1.25 \pm 1.00 \mathrm{~mm})$. The bone loss after 8 months for the allografts was spiked to $1.83 \pm 0.42 \mathrm{~mm}$ as compared with the xenografts $1.37 \pm 1.12 \mathrm{~mm}$ with no statistically significant difference $(p>0.05)$.

Conclusion: Both allografts and xenografts present comparable crestal bone level changes around dental implants when simultaneously placed during implant placement surgery.

Clinical significance: Both allografts and xenografts are suitable for the preservation of the alveolar ridge regarding crestal bone level changes. Selection of allografts and xenografts may not be carried out based upon the crestal bone level changes.

Keywords: Bone augmentation, Bone level changes, Dental implant success, Implant osteotomy.

The Journal of Contemporary Dental Practice (2021): 10.5005/jp-journals-10024-3195
\end{abstract}

\section{INTRODUCTION}

The outcome of the implant therapy significantly depends on the availability of a sufficient bone volume to support the implants in their optimum position. Alveolar bone atrophy and dimensional changes following dental extraction usually present a compromised clinical situation for the dental implants. ${ }^{1,2}$ Overall healing process after the tooth extraction leads to decrease in the alveolar ridge dimensions almost up to an average of $50 \%$ of its original size. ${ }^{3}$ This resorption pattern is particularly rapid in the first 3-6 months, followed by gradual reduction thereafter, throughout life. This bone resorption significantly reduces the possibility of dental implant therapy and creates both functional and esthetic issues during prosthetic rehabilitation. Based on the understanding of inevitable physiological bone resorption, alveolar ridge preservation procedures (ARP) with the application of various bone-grafting materials were developed to control the crestal bone resorption. The bone graft types include autograft, allograft, xenograft, and alloplastic grafts. Numerous studies have been performed to determine the clinical behavior of different graft materials with the allografts and xenografts being commonly used in implant therapy. ${ }^{3-7}$ The allografts are tissue grafts derived from another person other than person receiving the graft and are harvested from cadavers. ${ }^{8}$ They are classically referred to as having osteoinductive capabilities as well as quick resorption. ${ }^{3}$ The xenografts are taken from a species other than the human, for instance bovine. ${ }^{8}$ They are used as a natural calcified matrix by complete removal of the organic components to avoid any transmission
${ }^{1}$ School of Dentistry, International Medical University, Kuala Lumpur, Malaysia

${ }^{2}$ Department of Clinical Sciences, College of Dentistry, Ajman University, Ajman, United Arab Emirates

${ }^{3}$ Division of Restorative Dentistry, Department of Prosthodontics, School of Dentistry, International Medical University, Kuala Lumpur, Malaysia

Corresponding Author: Pravinkumar G Patil, Division of Restorative Dentistry, Department of Prosthodontics, School of Dentistry, International Medical University, Kuala Lumpur, Malaysia, e-mail: pravinandsmita@yahoo.co.in

How to cite this article: Mae CX, Shetty NY, Patil PG. Radiographic Evaluation of Crestal Bone Level Changes for Allografts or Xenografts Placed during Implant Placement: A Retrospective Study. J Contemp Dent Pract 2021;22(10):1082-1086.

Source of support: Study supported completely by the International Medical University under the Special Study Module. Project ID: BDS I-01-2018 (09).

Conflict of interest: None

of pathogens as well as immunological reactions. The remaining inorganic components have the capability for osteoconduction to maintain the tissue volume. ${ }^{3}$ If the bone loss measured from baseline to 6 or 8 months is less than $2 \mathrm{~mm}$, the graft material is considered satisfactory and suitable for grafting at the implant sites. 
Performance of different bone-grafting materials in terms of initial crestal bone level changes is not studied extensively, even though it is one of the most critical elements in overall implant treatment success. Comparison between xenografts and allografts in ARP procedure may provide useful information to clinicians and researchers. The aim of this study was to evaluate initial peri-implant crestal bone level changes when the allografts and xenografts were placed simultaneously during the implant placement.

\section{Materials and Methods}

This retrospective study examined the intraoral periapical (IOPA) radiographs of the patients undergone the bone-grafting procedure followed by the dental implant placement in the clinic of authors' institution. The institutional ethical committee clearance has been obtained [Project ID: BDS I-01-2018 (09)]. The radiographic records of the patients treated between January 2015 and June 2018 were selected with age more than 18 years who undergone bonegrafting procedure for dental implants either with the allograft (Puros ${ }^{\circledR}$ Cortico-Cancellous Particulate Allograft; Zimmer Biomet) or the xenograft (Cerabone, Biotiss Dental). All implants evaluated in the study were of Nobel Active (Nobel Biocare).

\section{Inclusion and Exclusion Criteria}

Medically compromised patients or patients who undergone additional grafting procedure or delayed implant placement were excluded from the study. The patients with distorted or blurred IOPA radiographs or absence of either of the required IOPA radiographs were also excluded from the study. The radiographs showing more than $1.5 \mathrm{~mm}$ of discrepancy in overall length of the implant appearing in the IOPA radiograph at different time points were also excluded to avoid significant measurement errors. Patients fulfilling the aforementioned criteria were divided and tabled based on the bone grafts they received-alloplastic, autogenous, allografts, and xenografts. Only allografts and xenografts were included in this study. Sample size $(n=77)$ was calculated based upon a pool of patients visiting the oral health center with $5 \%$ margin of error and $95 \%$ confidence interval and at the power of $80 \%$ by using an online sample size calculator (Raosoft Inc).

The IOPA radiographs were evaluated at four time intervals: (1) preoperative IOPA before surgery to evaluate the preoperative host bone level (Fig. 1A); (2) postoperative IOPA obtained immediately after surgery, which acts as the baseline (Fig. 1B); (3) after 3 months of surgery (Fig. 1C); and (4) after 6 or 8 months after surgery (Fig. 1D). The IOPA radiographs of the third recall visit were found to be either after 6 or 8 months after surgery, which were included in the study and divided into two subgroups. The measurements were carried out by taking the average level of the mesial and distal alveolar crestal bone from the neck of the implants to the topmost part of the bone graft material using the computer software program (Eigentool; Henry Ford) (Figs 1B to D). To calculate the alveolar crestal bone level changes around the implants, the extent of bone
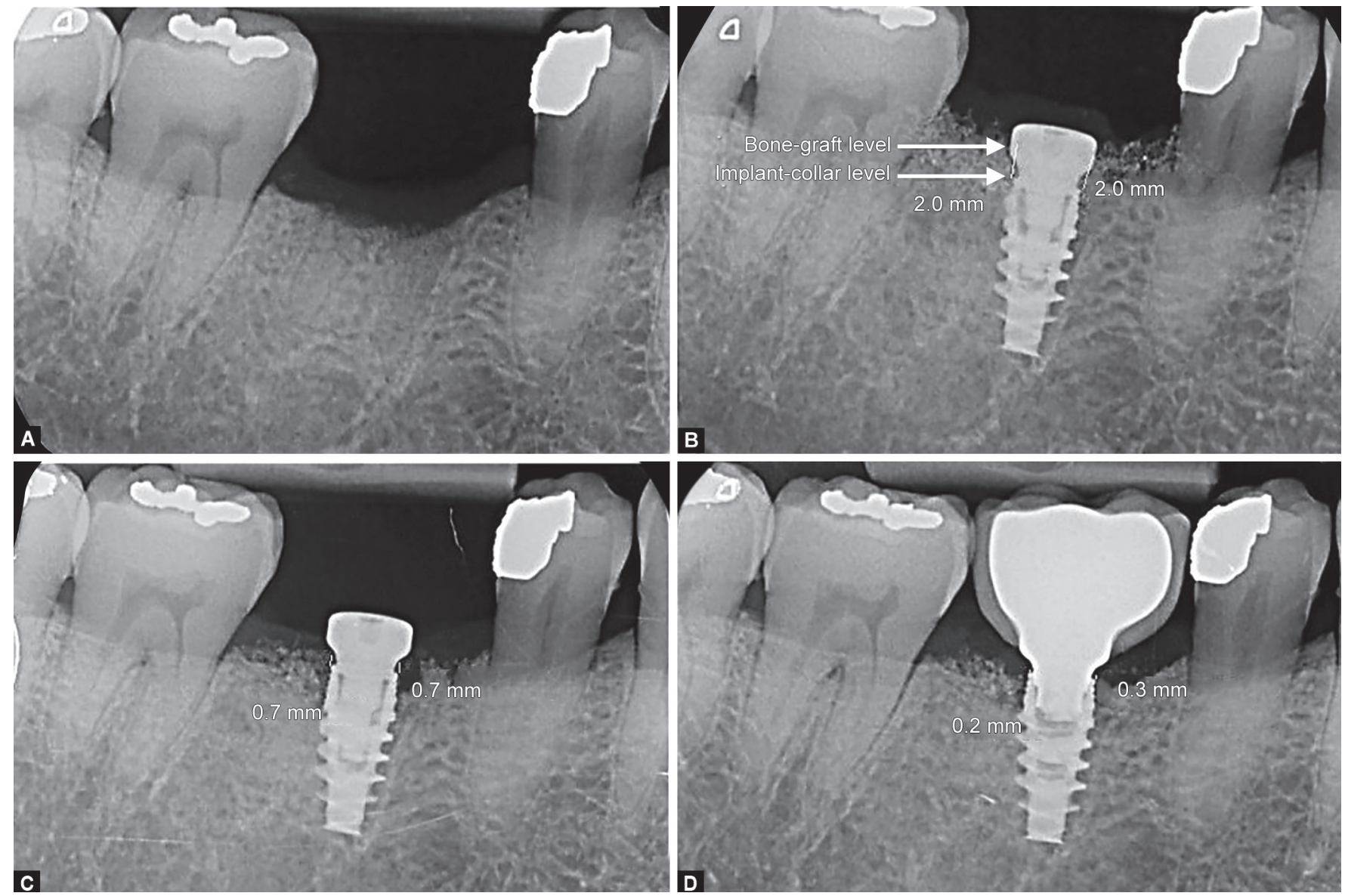

Figs 1A to D: (A) Preoperative IOPA; (B) Immediate postoperative IOPA. Note the level of measurements taken from crestal bone level to implant collar on both sides of implant; (C) After 3 months IOPA; (D) After 6 months IOPA 
resorption was measured by comparing radiographs obtained immediately after the surgery (baseline) to the IOPA acquired after 3 months and 6 or 8 months.

There were 77 implant sites selected from a total of 44 patients: total 59 implant sites for xenografts and 18 implant sites for allografts. All values were taken at the baseline, after 3 months of surgery, and after 6 or 8 months of surgery. The final time interval was further categorized into after 6 months and after 8 months of surgery.

The SPSS software version 23.0 (SPSS Inc; IBM) was used for data analysis. The changes in the marginal bone resorption and residual alveolar crestal bone height among both the groups were evaluated using a one-way analysis of variance (ANOVA), followed by post hoc Tukey Honestly Significant Difference (HSD) test with a significance level of $5 \%$.

\section{Results}

The mean differences in the alveolar bone resorption between baseline and postsurgical implant therapy with both bone graft materials are presented in Table 1. The alveolar bone loss at crestal region at the time of placement of bone grafts was $-1.85 \pm 1.26 \mathrm{~mm}$ at the xenograft sites and $-1.75 \pm 1.51 \mathrm{~mm}$ at allograft sites $(p=0.791$ ) (Table 1). At the time of reentry of 3 months, the bone dimension was $1.17 \pm 0.83 \mathrm{~mm}$ for the xenografts and $1.00 \pm 1.14$ for the allografts $(p=0.523)$. At the final reentry, bone-grafted sites were divided into 6 and 8 months postoperative visits. After 3 months, the allografts showed lesser bone resorption $(0.9 \pm 0.52 \mathrm{~mm})$ as compared with xenografts $(1.25 \pm 1.00 \mathrm{~mm})$ (Fig. 2). Interestingly, the bone loss after 8 months for the allografts was spiked to $1.83 \pm 0.42 \mathrm{~mm}$ compared with the xenografts $1.37 \pm 1.12 \mathrm{~mm}$ with no statistically significant difference level $(p>0.05)$.

Both allografts and xenografts showed loss of alveolar ridge height within the acceptable range of 1-2 $\mathrm{mm}$. The crestal bone loss after 3,6 , and 8 months were $1.0,0.9$, and $1.8 \mathrm{~mm}$ for allografts and $1.2,1.3$, and $1.4 \mathrm{~mm}$ for the xenografts.

\section{Discussion}

The objective of ridge preservation procedures using bone grafts is to prevent the atrophy of alveolar ridge as well as to preserve sufficient bone volume in order to promote the dental implant placement in prosthetically acceptable site or to preserve an admissible ridge contour for the purpose of esthetic concerns. This retrospective study evaluates the effectiveness of bone graft materials used in implant dentistry namely allograft and xenograft. Crestal bone level changes are one of the important parameters to evaluate the efficiency of any graft material used in dental implant treatment. The result of the present study showed bone loss even after the use of allograft or xenograft materials, which was predicted and consistent with several studies. ${ }^{3,9-12}$ The measurements were done with digital software with $0.1 \mathrm{~mm}$ of the least count to minimize the measurement errors.

It is well established that resorption of alveolar bone walls after extraction may happen in two parts. The first part involves the resorption of bundle bone as well as the formation of fibrous bone, resulting in vertical reduction of the alveolar ridge. ${ }^{1-3}$ The second part is a horizontal reduction of the alveolar ridge due to buccal bone walls resorption. ${ }^{2}$ An assessment of the changes in the dimension of postextraction the alveolar hard and soft tissue was made in a systematic review. The review concluded that the horizontal resorption of the alveolar bone at 6 month postextraction was $29-63 \%(2.46-4.56 \mathrm{~mm})$, while the vertical resorption was $11-22 \%(0.8-1.5 \mathrm{~mm})$. The expected outcome of the bone remodeling process is a narrower and shorter ridge..$^{13,14}$

Several studies showed a positive result of ARP for limiting both horizontal and vertical bone dimensional loss compared to a spontaneous socket healing of the alveolar ridge. ${ }^{3}$ In a recent study, ARP performed in the posterior maxilla with a combination of allograft and collagen membrane resulted in $1.0 \mathrm{~mm}$ crestal height reduction and in approximately $2.5 \mathrm{~mm}$ loss of alveolar ridge width. This volumetric contraction was lower than the one observed in extraction sites of the same area after spontaneous healing. ${ }^{15}$ lasella et al. showed that preservation of alveolar ridge using freeze-dried bone allograft and collagen membrane resulted in less alveolar ridge loss compared to extraction alone $(1.2 \pm 0.9 \mathrm{vs}$ $2.6 \pm 2.3 \mathrm{~mm}$ ). Both allografts and xenografts partially preserved the width and the interproximal bone height of the alveolar ridge. ${ }^{12}$

Although a decrease in the alveolar ridge height was observed in both groups, both bone graft materials were considered satisfactory in terms of allowing the placement of dental implants. From baseline up to 6 and 8 months interval, the loss of bone was within the normal range of 1-2 $\mathrm{mm}$ for the sites treated with both xenografts

Table 1: Mean difference of crestal bone level changes at dental implant sites

\begin{tabular}{llcccccc}
\hline & & & \multicolumn{3}{c}{$95 \%$ CI } & & \\
\cline { 5 - 6 } & & $N$ & Mean & Lower bound & Upperbound & Std. deviation & p value \\
\hline Baseline & Xenograft & 59 & -1.847 & -2.177 & -1.518 & 1.2639 & \\
& Allograft & 18 & -1.753 & -2.506 & -0.999 & 1.5155 & 0.791 \\
& Total & 77 & -1.825 & -2.124 & -1.526 & 1.317 & \\
After 3 months & Xenograft & 59 & 1.165 & 0.949 & 1.381 & 0.8287 & \\
& Allograft & 18 & 1.008 & 0.441 & 1.576 & 1.1407 & 0.523 \\
& Total & 77 & 1.129 & 0.923 & 1.334 & 0.9053 & \\
After 6 months & Xenograft & 34 & 1.254 & 0.903 & 1.606 & 1.0069 & \\
& Allograft & 7 & 0.9 & 0.418 & 1.382 & 0.5212 & 0.374 \\
& Total & 41 & 1.194 & 0.895 & 1.493 & 0.9463 & \\
After 8 months & Xenograft & 25 & 1.37 & 0.908 & 1.832 & 1.1188 & \\
& Allograft & 11 & 1.827 & 1.545 & 2.11 & 0.4203 & 0.200 \\
& Total & 36 & 1.51 & 1.179 & 1.84 & 0.9769 & \\
\hline
\end{tabular}

$\mathrm{Cl}$, confidence interval for mean; SD, standard deviation 


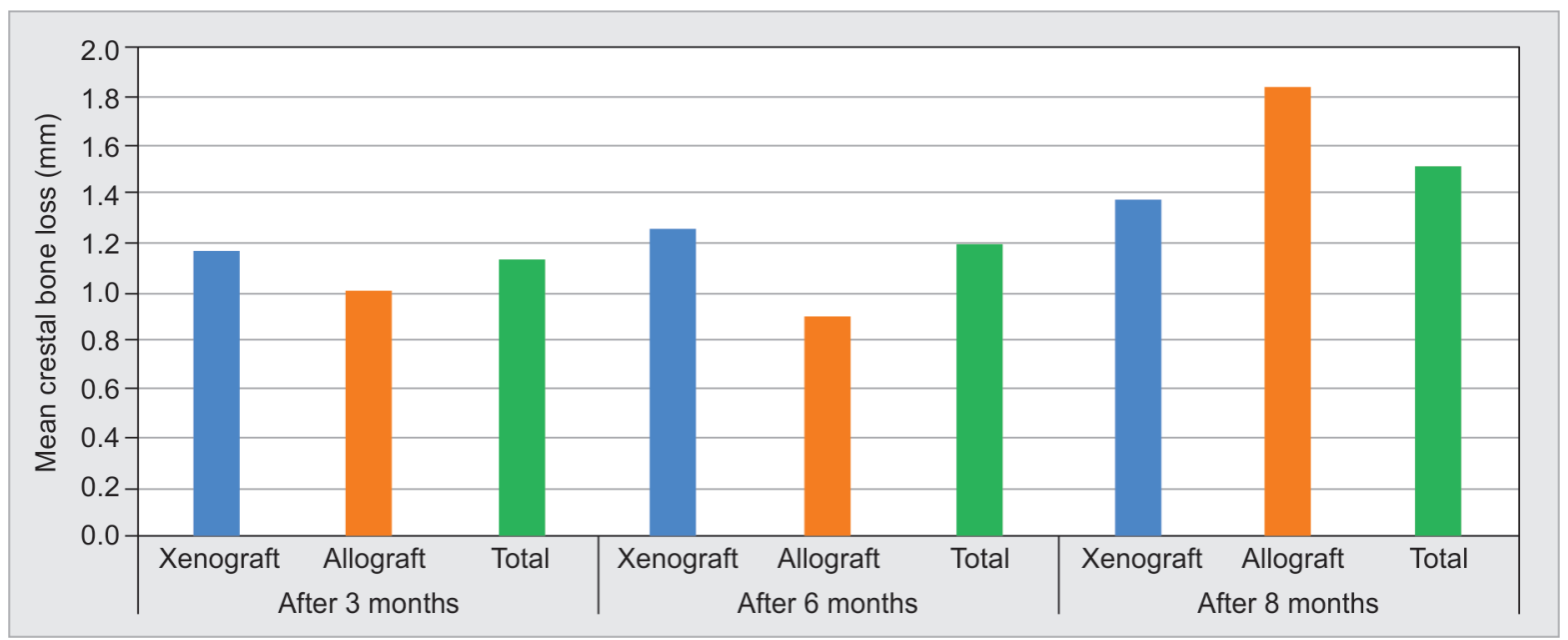

Fig. 2: Graph indicating mean difference of alveolar bone resorption at dental implant sites after 3, 6, and 8 months

and allografts, which were in agreement with Ten Heggeler et al. ${ }^{16}$ In a clinical study by Mendez et al, healing of allografts and xenografts for alveolar ridge preservation was compared by measuring the vertical and horizontal bone dimensions at baseline and 6 months later. $^{3}$ The results at the allografts sites $(-0.6-0.5 \mathrm{~mm})$ appeared to have less resorption compared to that at the xenograft sites $(-1.1-(-0.4) \mathrm{mm})$. This has corroborated the results of the present study when the measurement was taken after 6 months: allograft sites $(0.42-1.38 \mathrm{~mm})$ and xenograft sites $(0.90-1.61 \mathrm{~mm})$. However, at 8 months interval, allograft sites $(1.55-2.11 \mathrm{~mm})$ showed higher resorption than xenograft sites $(0.91-1.83 \mathrm{~mm})$.

Lee et al. ${ }^{17}$ concluded in a histomorphometric study that DBBM showed consistent osteoconductive effects, demonstrating significantly greater mean areas $(p<0.05)$ of new bone and residual graft particles, with less remaining fibrous tissue than irradiated cancellous allograft (ICA) or solvent-dehydrated allograft (SDA). ${ }^{17}$ The results also showed that ICA and SDA were transformed into new bone rapidly through osteoinduction process; however, they were gradually surrounded by more cellular and vascular fibrous tissues. Vance et al. ${ }^{4}$ have shown that ridge preservation using an intrasocket graft of carboxymethylcellulose and CaS with demineralized freeze-dried bone allograft covered by a CaS barrier produced a significantly higher percentage of vital bone fill at 4 months than bovine-derived xenograft (BDX) with a collagen membrane, while BDX with a collagen membrane was associated with more residual graft particles and less vital bone. ${ }^{4}$

However, the study had limitations. There were only 77 implantgrafted sites finally included under the inclusion/exclusion criteria. There was also a bias toward the number of patients receiving xenografts than allografts. As the follow-up times for the patients were different, the final time intervals had to be divided further into 6 and 8 months postoperative recall period, which could have influenced the results of this study. Radiographs taken may be of different angulations and positions at different time intervals, which would also affect the results of this study. Future direction of research should include a larger sample size with a more uniform number of sites for each group. The follow-up time for each patient should be standardized as well to provide more conclusive evidence. As autografts and alloplastic grafts were not included in this study and future studies should compare the efficacy of these bone-grafting materials as well. The radiographs showing more than $1.5 \mathrm{~mm}$ of discrepancy in overall length of the implant appearing in the IOPA radiograph were not included in the research to minimize the measurement errors in this retrospective study. Measurements can be normalized with the formula given by Patil et al. ${ }^{18}$ Clinical point of view, both allografts and xenografts are suitable for the preservation of the alveolar ridge in regard to crestal bone level changes. Selection of allografts and xenografts may not be carried out based upon the crestal bone level changes. Other parameters like quality and speed of the tissue healing, bone density of the healed sites, and implant stability can be evaluated to evaluate efficacy/outcome of graft materials in future.

\section{Conclusion}

Within the limitations of this study, following conclusion can be drawn. The crestal bone level changes are comparable with both allografts and xenografts, as no statistically significant differences were found. Hence, from a clinical standpoint, both allografts and xenografts are suitable for the preservation of the alveolar ridge regarding the crestal bone level changes.

\section{References}

1. Qabbani AA, Kawas SA, Enezei H, et al. Biomechanical and radiological assessment of immediate implants for alveolar ridge preservation. Dent Res J 2018;15(6):420-429. PMID: 30534170; PMCID: PMC6243806.

2. Natto Z, Yaghmoor W, Bannuru R, et al. Identification and efficacy ranking of allograft and xenograft for extraction and ridge preservation procedures. Int J Periodontics Restorative Dent 2017;37(5):e253-e260. DOI: 10.11607/prd.3323.

3. Méndez CAS, Lang NP, Caneva $M$, et al. Comparison of allografts and xenografts used for alveolar ridge preservation. A clinical and histomorphometric RCT in humans Clin Implant Dent Relat Res 2017;19(4):608-615. DOI: 10.1111/cid.12490.

4. Vance GS, Greenwell H, Miller RL, et al. Comparison of an allograft in an experimental putty carrier and a bovine-derived xenograft used in ridge preservation: a clinical and histologic study in humans. Int J Oral Maxillofac Implants 2004;19(4):491-497. PMID: 15346745.

5. Becker W, Becker BE, Caffesse R. A comparison of demineralized freeze-dried bone and autologous bone to induce bone formation 
in human extraction sockets. J Periodontol 1994;65(12):1128-1133. DOI: 10.1902/jop.1994.65.12.1128.

6. Dies F, Etienne D, Abboud NB, et al. Bone regeneration in extraction sites after immediate placement of an e-PTFE membrane with or without a biomaterial. A report on 12 consecutive cases. Clin Oral Implants Res 1996;7(3):277-285. DOI: 10.1034/j.1600-0501.1996.070310.x.

7. Kangwannarongkul T, Subbalekha K, Vivatbutsiri $P$, et al. Gene expression and microcomputed tomography analysis of grafted bone using deproteinized bovine bone and freeze-dried human bone. Int J Oral Maxillofac Implants 2018;33:541-548. DOI: 10.11607/ jomi.6234.

8. Kumar P, Fathima G, Vinitha B. Bone grafts in dentistry. J Pharm Bioallied Sci 2013;5(Suppl. 1):S125-S127. DOI: 10.4103/09757406.113312.

9. Iasella JM, Greenwell H, Miller RL, et al. Ridge preservation with freeze-dried bone allograft and a collagen membrane compared to extraction alone for implant site development: a clinical and histologic study in humans. J Periodontol 2003;74(7):990-999. DOI: 10.1902/jop.2003.74.7.990.

10. Babaei M, Sadeghi R, Miremadi S, et al. A randomized controlled evaluation of alveolar ridge preservation following tooth extraction using deproteinized bovine bone material and demineralised freeze-dried bone allograft. Dental Res J 2016;13(2):151-159. DOI: 10.4103/1735-3327.178202.

11. Nart J, Barallat L, Jimenez D, et al. Radiographic and histological evaluation of deproteinized bovine bone mineral vs. deproteinized bovine bone mineral with $10 \%$ collagen in ridge preservation. A randomized controlled clinical trial. Clin Oral Implants Res 2016;28(7):840-848. DOI: 10.1111/clr.12889.
12. Mardas N, Chadha V, Donos N. Alveolar ridge preservation with guided bone regeneration and a synthetic bone substitute or a bovine-derived xenograft: a randomized, controlled clinical trial. Clin Oral Implants Res 2010;21(7):688-698. DOI: 10.1111/j.16000501.2010.01918.x.

13. Tan W, Lang N, Wong T, et al. A systematic review of post-extractional alveolar hard and soft tissue dimensional changes in humans. Clin Oral Implants Res 2012;23(Suppl 5):1-21. DOI: 10.1111/j.16000501.2011.02375.x.

14. Darby I, Chen ST, Buser D. Ridge preservation techniques for implant therapy. Int J Oral Maxillofac Implants 2009;24(Suppl.):260-271. PMID: 19885449.

15. Lombardi T, Bernardello F, Berton F, et al. Efficacy of alveolar ridge preservation after maxillary molar extraction in reducing crestal bone resorption and sinus pneumatization: a multicenter prospective case-control study. Biomed Res Int 2018:9352130. DOI: 10.1155/2018/9352130.

16. Ten Heggeler JM, Slot DE, Van der Weijden GA. Effect of socket preservation therapies following tooth extraction in non-molar regions in humans: a systematic review. Clin Oral Implants Res 2010;22(8): 779-788. DOI: 10.1111/j.1600-0501.2010.02064.x.

17. Lee DW, Pi SH, Lee SK, et al. Comparative histomorphometric analysis of extraction sockets healing implanted with bovine xenografts, irradiated cancellous allografts, and solvent-dehydrated allografts in humans. Int J Oral Maxillofac Implants 2009;24(4):609-615. PMID: 19885400

18. Patil PG, Nimbalkar-Patil S. A radiographic measurement technique for crest bone changes related to dental implants. J Prosthet Dent 2015;113(4):350-351. DOI: 10.1016/j.prosdent.2014.11.002. 